\title{
Liquid-Liquid Equilibrium Data and Process Simulation for Separating the Mixture of Decanol + Undecanol + Tetradecane + Pentadecane
}

\author{
Shenfeng Yuan, Genlai Bao, Hong Yin*, and Zhirong Chen \\ College of Chemical and Biological Engineering, Zhejiang Provincial Key Laboratory of Advanced Chemical \\ Engineering Manufacture Technology, Zhejiang University, Hangzhou 310027, P. R. China \\ * Corresponding author: Hong Yin; yinh@ zju.edu.cn
}

\section{Determining uncertainties}

a. The temperature uncertainty

Affected by room temperature, the temperature of the super thermostatic water bath fluctuates. As a result, the maximum temperature fluctuation inside the liquid-liquid equilibrium kettle is $0.05-0.1 \mathrm{~K}$, therefore, the temperature uncertainty is set to $0.1 \mathrm{~K}$.

b. The pressure uncertainty

In the liquid-liquid experiments, the maximum pressure fluctuation reached $1.0 \mathrm{kPa}$, which was far larger than the accuracy of pressure gauge $(0.1 \mathrm{kPa})$. Therefore, ' $101.3 \pm 1.0 \mathrm{kPa}$ ' was used to describe the pressure.

c. The mass fraction uncertainty

Considering the mass fraction covers a wide range, the relative uncertainty $U_{r}$ should be a better descriptor. The equations of relative uncertainty $U_{r}$ are as follows: 


$$
\begin{gathered}
\overline{w_{l}}=\frac{\sum_{k=1}^{n} w_{i, k}}{n} \\
u_{A}\left(w_{i}\right)=S\left(\overline{w_{l}}\right)=\sqrt{\frac{1}{n(n-1)} \sum_{k=1}^{n}\left(w_{i, k}-\overline{w_{l}}\right)^{2}} \\
U_{r}=\frac{u_{A}\left(w_{i}\right)}{\overline{w_{l}}}
\end{gathered}
$$

Where, $w_{i, k}$ is the mass fraction of component $i$ for one sample measured under the same gas chromatography conditions, $\overline{w_{l}}$ is the average mass fraction, and $n$ is the number of analysis.

\section{Experimental results on lab scale}

Table 1. The experimental results on lab scale

\begin{tabular}{cccccccccc} 
stream & $\mathrm{F} 101$ & $\mathrm{~S} 101$ & $\mathrm{~S} 102$ & $\mathrm{~S} 103$ & $101 \mathrm{E}$ & $101 \mathrm{R}$ & $102 \mathrm{E}$ & $102 \mathrm{R}$ & $103 \mathrm{E}$ \\
$\begin{array}{c}\text { temperature }{ }^{\circ} \mathrm{C} \\
\text { mass fraction }\end{array}$ & 25 & 25 & 35 & 25 & 25 & 25 & 35 & 35 & 25 \\
ethanol & 0 & 0.8 & 0 & 0 & 0.6806 & 0.0254 & 0.0201 & 0.7451 & 0.0583 \\
decanol & 0.2976 & 0 & 0 & 0 & 0.0658 & trace & 0.0184 & 0.0469 & trace \\
undecanol & 0.2518 & 0 & 0 & 0 & 0.0537 & trace & 0.0167 & 0.0365 & trace \\
tetradecane & 0.2572 & 0 & 0 & 0 & 0.0219 & 0.5113 & 0.0455 & trace & trace \\
pentadecane & 0.1934 & 0 & 0 & 0 & 0.0144 & 0.4633 & 0.0283 & trace & trace \\
water & 0 & 0.2 & 0 & 1 & 0.1636 & trace & 0.0007 & 0.1584 & 0.9417 \\
octadecane & 0 & 0 & 1 & 0 & trace & trace & 0.8703 & 0.0131 & trace \\
stream & $103 \mathrm{R}$ & $105 \mathrm{D}$ & $105 \mathrm{~W}$ & $106 \mathrm{D}$ & $106 \mathrm{~W}$ & $\mathrm{~V} 101-\mathrm{O}$ & $\mathrm{V} 101-\mathrm{W}$ & $108 \mathrm{D}$ & $108 \mathrm{~W}$ \\
temperature ${ }^{\circ} \mathrm{C}$ & 25 & 78.20 & 101.13 & 80.43 & 203.05 & 25 & 25 & 125.49 & 197.23 \\
mass fraction & & & & & & & & & \\
ethanol & 0.0049 & 0.9463 & trace & 0.0549 & trace & 0.0005 & trace & 0.0008 & trace \\
decanol & trace & trace & 0.2148 & 0.1587 & trace & 0.4526 & trace & 0.5236 & trace \\
undecanol & trace & trace & 0.1586 & 0.1440 & trace & 0.4267 & trace & 0.4704 & trace \\
tetradecane & 0.5008 & trace & trace & 0.3924 & trace & 0.0006 & trace & 0.0008 & trace \\
pentadecane & 0.4943 & trace & trace & 0.2440 & trace & 0.0004 & trace & 0.0005 & trace \\
water & trace & 0.0537 & 0.5631 & 0.0060 & trace & 0.0265 & 1.0000 & 0.0039 & trace \\
octadecane & trace & trace & 0.0635 & trace & 1.0000 & 0.0927 & trace & trace & 1.0000 \\
\hline
\end{tabular}

